\title{
AUTMOD3: AN AUTOMATIC 3D MODULARIZATION SYSTEM*
}

\author{
R. Diez, M. Abderrahim, V. M. Padrón, L. Celorrio, J. M. Pastor \\ and C. Balaguer
}

\author{
Department of Electrical Engineering, Electronics and Automation \\ Universidad Carlos III de Madrid \\ C/Butarque, 15, 28911 Leganés, Madrid (Spain) \\ e-mail: \{rdiez, mohamed, balaguer,vmpadron\}@ing.uc3m.es
}

\begin{abstract}
This paper presents the new automatic system called AUTMOD3 for automatic design of modular buildings. The system permits to obtain in an automatic way, and with guided assistance the dimensions and position of the 3D and/or 2D modules that are needed to construct a building starting from the traditional architectural design. The system is based on the well-known CAD system commonly used by designers. Specially developed software modules, menus and libraries are linked to the CAD system. A library of several types of modules assists and guides the designer through the process. It contains structural 3D modules, 2D facade modules, 3D roof modules, etc. The selection of the modules will be carried out responding to several production criteria: minimum number of modules, minimum number of different modules, module size limitation due to pre-fabrication process, etc. Other criteria are also taken into account: module size limitation due to transportation restriction, limitation due to on-site assembly, etc. The proposed modularization system does take into consideration only technical aspects of the designed building. Nevertheless, future developments will include some aesthetic aspects.
\end{abstract}

Keywords: architectural design, modular design, design automation.

\section{INTRODUCTION}

Traditional methods on house-building are usually based on manual techniques which are slow and expensive. The benefits of construction industries can be improved by using advanced manufacturing systems. These systems will increase the quality and the customer satisfaction. Nowadays, other industries take advantage of automatic production and assembly processes.

Despite that the research to develop new construction techniques has been important during the past few years [2], there is still a long distance between the construction industry and other industries. A change in the construction methods and the acceptance of prefabrication technologies are essential for the construction industry to reach a successful widespread in the market.

One of the construction industry main problems is the exchange of information between the different stages (design, planning, transport, erection, maintenance, etc.). The integration of activities should be an objective to increase benefits $[4,9]$.

This work is focused on the design stage. It presents a system, which adapts and links the methods of traditional architectural design with new construction methods.

The objective is to calculate the $3 \mathrm{D}$ modules and 2D panels, which are needed to construct a block of flats starting from the traditional architectural design [3]. These panels and modules will be pre-fabricated in a factory and will be transported to the site for their automatic assembly with robots.

We have taken as a starting-point the study of an apartment block, although the method could be extended to other types of buildings like row of terraced houses, detached houses, office blocks, etc. Figure 1 shows an example of the apartment block, which has been employed to test the system. The upper figure is a model representation of the building. The real building, bottom figure, has been constructed by the Spanish company Dragados, S.A. in Boadilla del Monte (Madrid).

Partially supported by FutureHome project (BRITE n ER4-29671) and PAUTA project 1691/91 

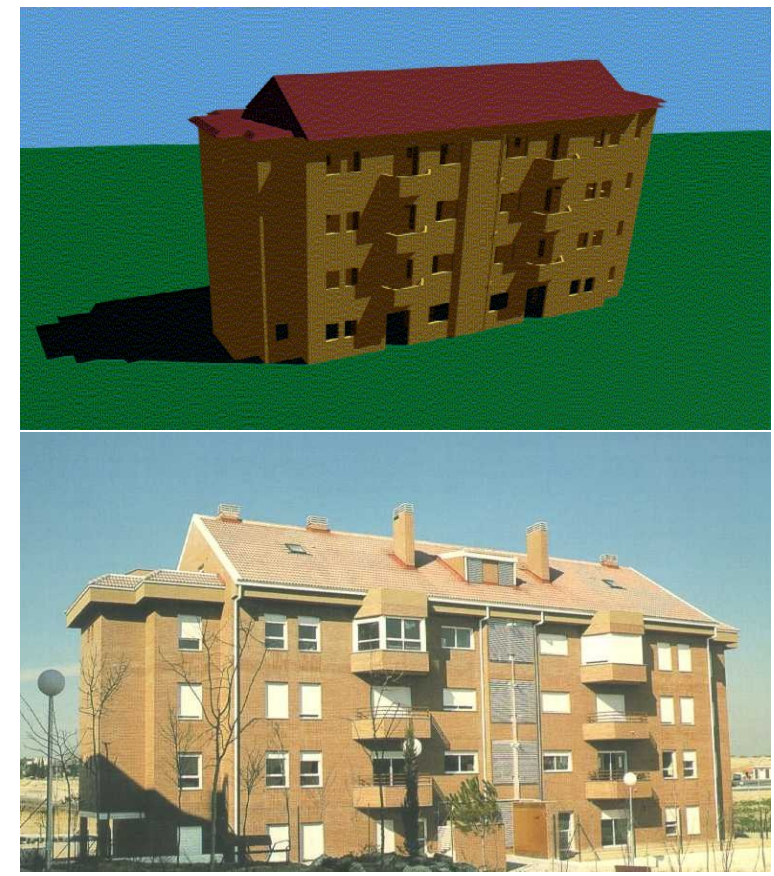

Figure 1. Example: model representation and real building (Courtesy of Dragados S.A.)

The study has been divided into 2 parts. On the one hand, we have the structural modules calculation, which forms the interior (these modules are threedimensional). On the other, we have the twodimensional panels' calculation, which form the external facade [1], [5].

\section{SYSTEM ARCHITECTURE}

The automatic modularization system is formed by a set of applications or modules and a group of data libraries. A graphical interface integrates the

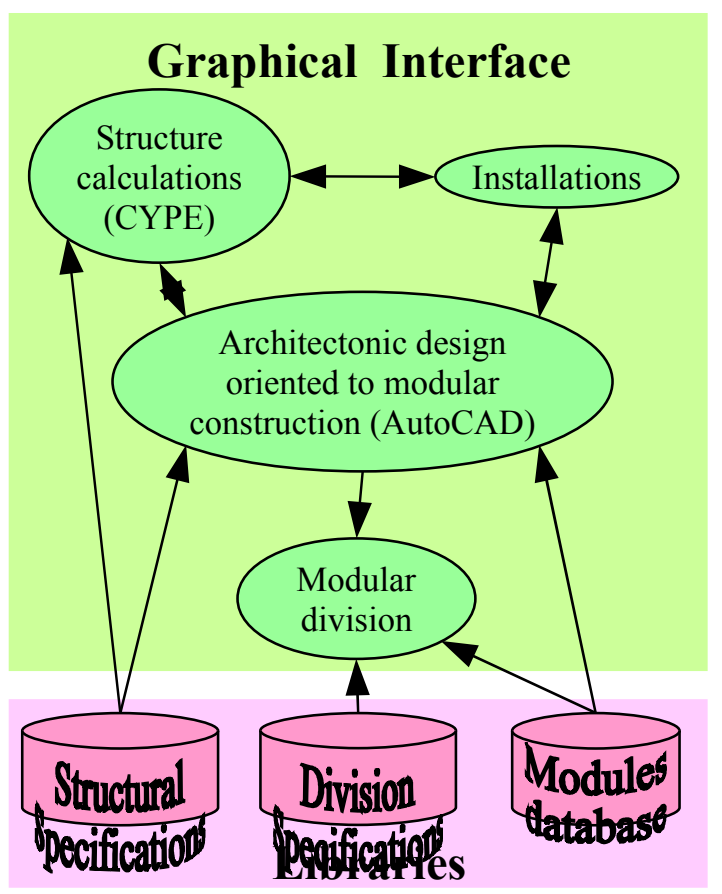

Figure 2. AUTMOD3 system architecture. different modules. Figure 2 shows a schematic representation of the system architecture.

AutoCAD has been selected as the main software package, because of its widespread use and because it incorporates several development tools. The chosen development tool for the implementation is the AutoCAD Runtime Extension (ObjectARX). A group of new functions has been created in order to enlarge and adapt AutoCAD to the problem specification. To facilitate the design, these additional software utilities are included in the AutoCAD menu bar.

The structure calculations are achieved by a finite element program (CYPE) which is compatible with the AutoCAD data files. Another module carries out the building installations calculation: heating, ventilation and air conditioning (HVAC).

The modular division is calculated with an application developed in $\mathrm{C}++$ with ObjectARX [8]. This module is executed from within AutoCAD. It takes data from the drawings performed in AutoCAD with the modifications made by the other modules.

The system includes a group of libraries, which contain the module characteristics, the construction criteria or the modular division specifications' information. These libraries are the following:

Modules database contains the modules data, their dimension parameters, their accessories, and other characteristics.

Structural specifications contain information about the building structure.

Division specifications contain criteria to calculate the number and type modules required to form a building, having as input the 2D architectural design.

\section{MODULAR DESIGN}

\subsection{Traditional design.}

The design process can be grouped in a small number of stages (see figure 3). With the specifications and requirements demanded by the client a first design is drawn. The details and accessories are added in the CAD system.

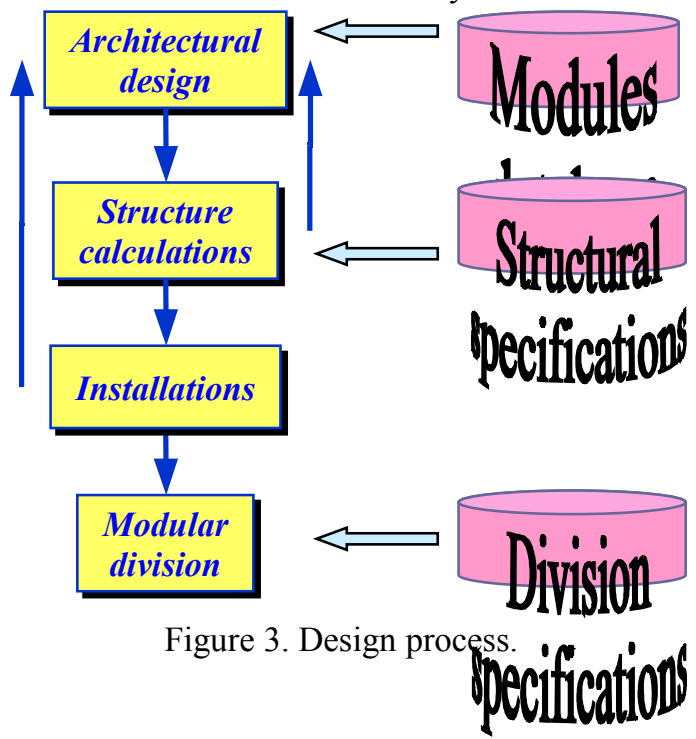


The next step is the structural calculation, to check that the structure resists and fulfils th existent norms. For this calculation specific pres are employed. If the structure does not resist a return

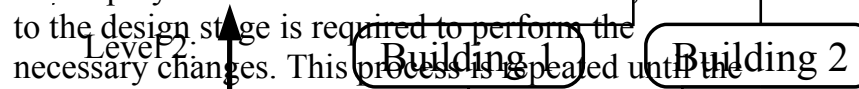
structure is acc ptable.

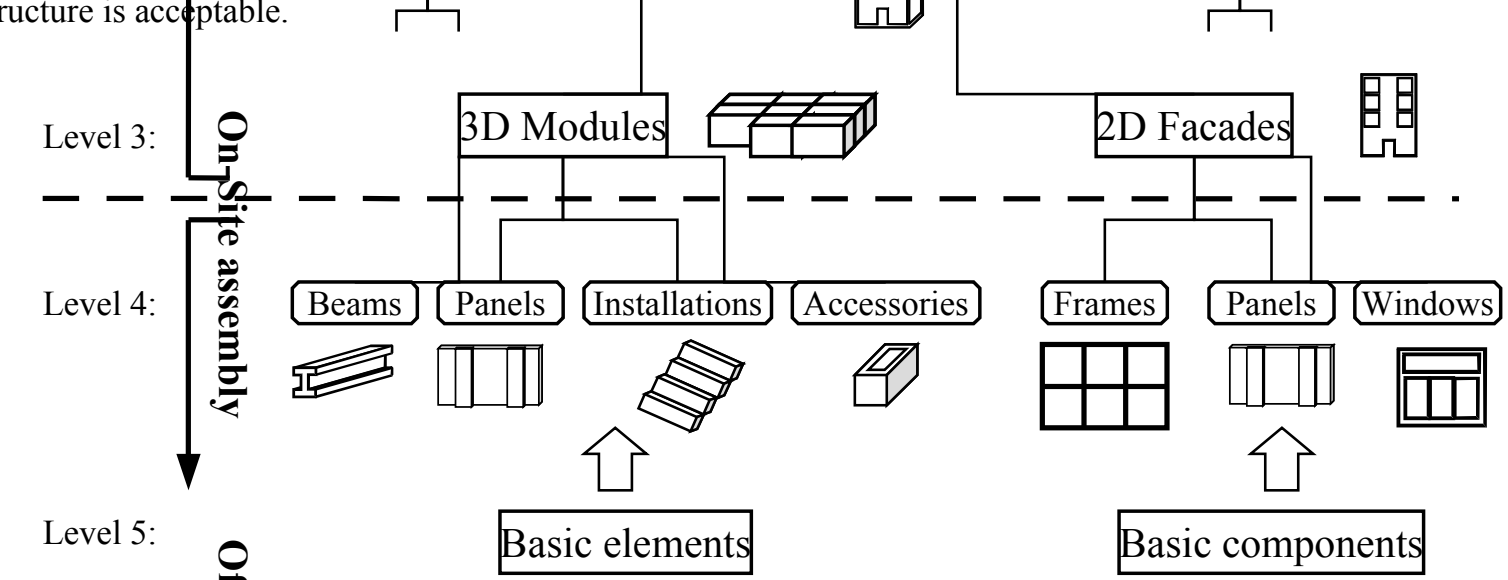

Figure 4. Tree structure database.

The following stage carried out is the housing installation calculation: heating, ventilation, air conditioning, etc. If any of these installations could not be placed appropriately or it does not fulfil the regulations, another return to the design module is performed to modify the drawing and repeat the process. Once this phase is completed, traditional design is finished.

\subsection{Automatic modular division.}

On the stages seen in the previous section the two-dimensional plans of the building are drawn. With the data from the drawing and some additional dimension (mainly heights of different elements), the application calculates the modules, which will constitute the building. In this calculation one must keep in mind a series of restrictions imposed by: the customer specifications, urban regulations (maximum number of floors, height, etc.), characteristics of the ground/land, economical factors, etc. Following the selection of the adequate division criteria the system is instructed to curry out the necessary calculation.

\section{AUTOMATIC 3D MODULARIZATION}

The modularization can be divided in two parts: on the one hand the calculation of the interior structure with three-dimensional modules and on the other, the external walls division in two-dimensional panels. The latter is dealt with in section 5 .
In both cases a modules' library is provided. A series of criterions for the modular calculation and a group of restrictions should be taken in consideration upon applying these criterions. Figure 4 shows the model data structure.

\subsection{Types of modules.}

Automation is related to the repetition of tasks, with big product series. The architectural design carries implicitly the idea of exclusivity. One could not make a group of different modules for each building. However, the available modules catalogue should be extensive enough to not limit the designers

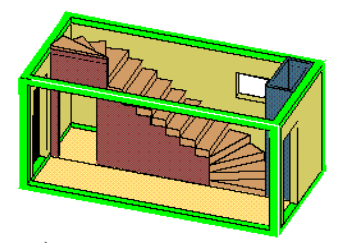

a)

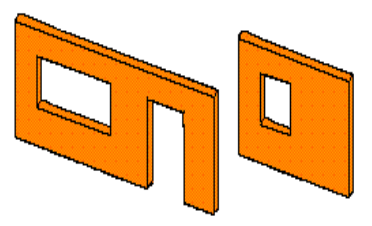

b) creativity. The best solution should be a compromise between both ideas.

Figure 5. a) 3D module, b) facade panels.

The built modules library is made up of threedimensional modules and two-dimensional panels. A distinction is made between structural and not structural modules. The modules dimensions vary in a finite range of measuring, with the result of a reduced number of different modules. The diversity 
is obtained with the additional elements and the materials employed to complete the installations.

The modules could be classified in 3 types: 3D modules, 2D panels and roofs. A fourth type, including special modules could be added. These special modules cover any constructive detail that could not be carried out with the basic group.

The three-dimensional modules will be structural. Their length will vary from 1.2 to $10.8 \mathrm{~m}$, while the width ranges from 1.2 to $2.4 \mathrm{~m}$ (with a $30 \mathrm{~cm}$. step). The typical height is $2.7 \mathrm{~m}$. With these modules we make the volumetric modules for kitchens, bath, stairways, etc. They will incorporate all the necessary installations. Figure 5 a) shows a three-dimensional module sample.

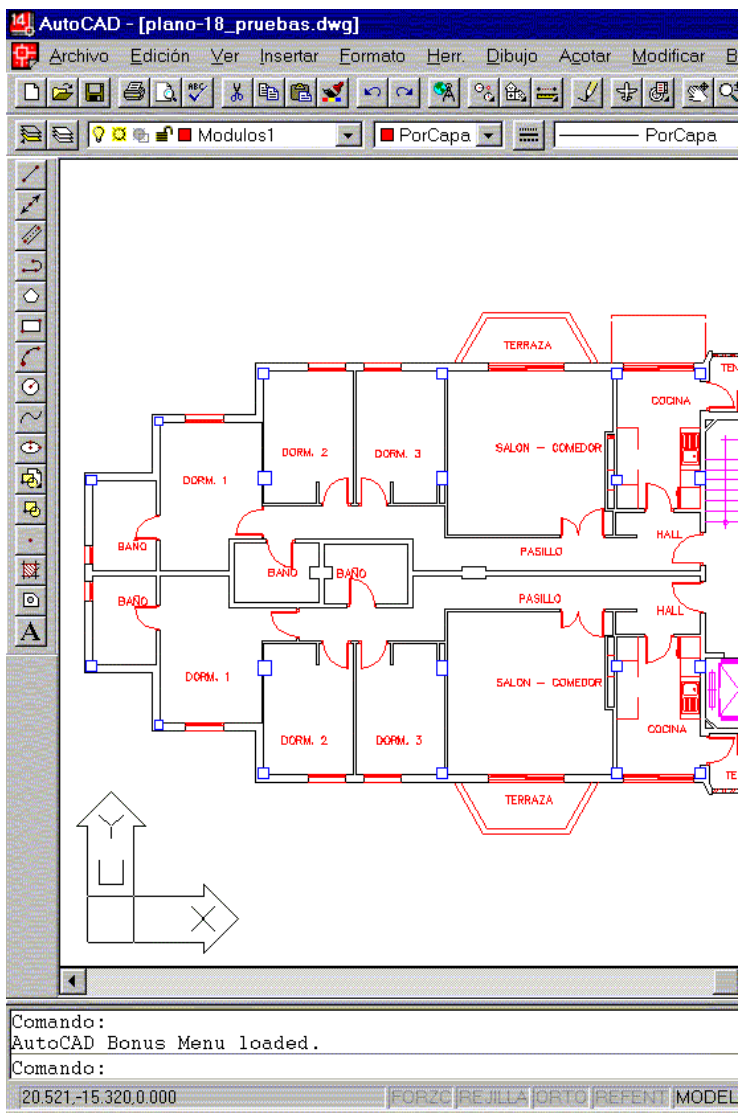

Figure 6. Drawing of the building.

The two-dimensional panels could be divided in structural and not structural panels, where the former is employed to complete the building structure. Their dimension will be up to $6 \mathrm{~m}$ of length and $2.7 \mathrm{~m}$ of height. The floor system panels have a maximum dimension of $6 \times 2.7 \mathrm{~m}$. The facades will be formed by no-structural panels (maximum dimensions of $6 \mathrm{x}$ $3 \mathrm{~m}$ ). Two facade panels are shown in figure $5 \mathrm{~b}$ ).

The roof modules are three-dimensional modules assembled from two-dimensional panels, in the fieldfactory. In this group other special modules like chimneys are included.

\subsection{Criteria of modular division}

For the modular division, we have established some criteria. The first is based on the building structure, that is, one must maintain the structural elements in their position (see figure 7). The second one is based on the room distribution; where an attempt is made to fit each room within a module (see figure 8).

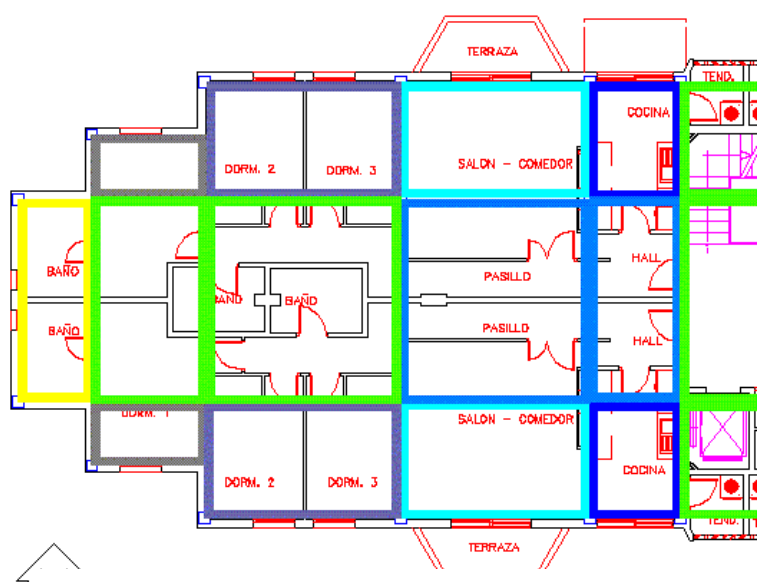

Figure 7. Modularization with criterion 1.

Other criteria are based on getting the minimum number of modules or the maximum number of identical modules, that is, using the minimum number of different modules. One could apply combinations of these methods attempting to take advantage of the best qualities offered by each method.

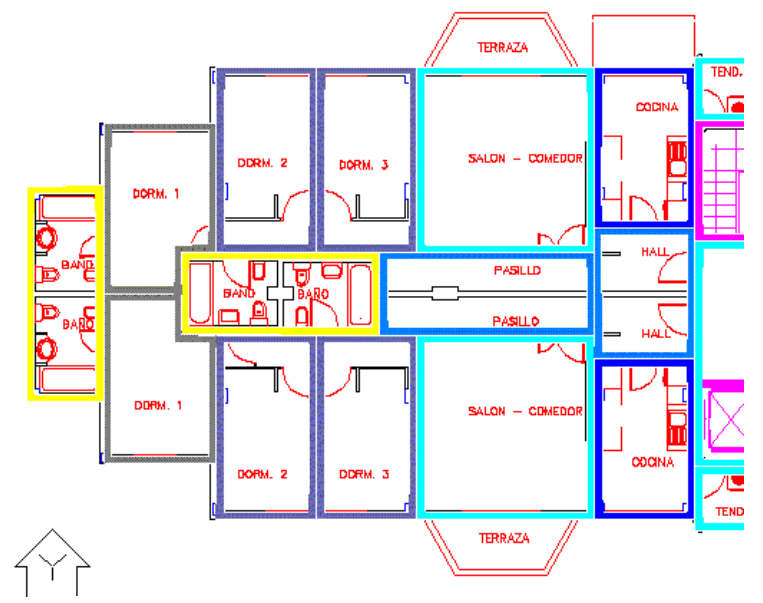

Figure 8. Modularization with criterion 2.

When applying these criterions one must consider a series of restrictions. For instance the presence of garages or other special installations forces the structural elements to maintain their original position. This impedes the application of some criteria. The land characteristics and the location are other factors to be considered. The transportation to the site is a factor that limits the maximum modules size. Finally one must consider economical factors and the client specifications. 


\subsection{Stages in the process of modular division}

For the modular division we start from a twodimensional drawing with the building plant (see figure 6). This drawing should contain additional information like the different elements height (roofs, doors, windows, etc.). The division process is performed in stages as follow:

- Data reading from the drawing that they will be in a well-known format.

- Data conversion to an adequate structure for their analysis. In order to facilitate these two first steps a set of recommendations will be followed.

- Information analysis, in order to extract the building characteristics: to identify the elements (beams, walls, doors, windows, ...), the rooms distribution (kitchens, bath, stairways, etc.).

- Application of the modular construction selected criterion, considering a group of rules and a series of restrictions.

- Modules listing obtained in an adequate format for their later treatment.

\section{FACADE PARTITION}

The second problem consists of calculating the 2D panels, which will form the facades [7].

\subsection{Facade panels restrictions}

It is necessary to consider process specifications to perform the automatic building facade generation. The process will observe the following restrictions:

The facade panels moulds could exceed neither the maximum, nor the minimum dimensions.

The building carpentry elements (doors, windows, etc.) will remain included in one panel.

Cuts should be located at a minimum distance from the prohibited zone borders. When it is not possible to avoid cutting forbidden zones, the process will continue, always respecting the previous rule of margin around the forbidden zones borders.

On one hand, it is necessary to keep in mind the tolerances of the mould construction and, on the other hand, the panel dilatation, which depends on the material used in their construction.

\subsection{Automatic facade partitions}

In the first step we divide the facade according to its external faces (see figure 9). Before partitioning, we analyse the facades geometry to extract their characteristics in a similar way to [6].

The facade generation process is divided in four phases that are executed consecutively: extracting of the facade main faces, calculation of horizontal divisions, calculation of forbidden zones and calculation of vertical divisions.
The obtaining of the horizontal cuts is based on the storeys height and form. Designers most followed rule to divide horizontally the facade is to take each floor intersection with the facade as division lines.

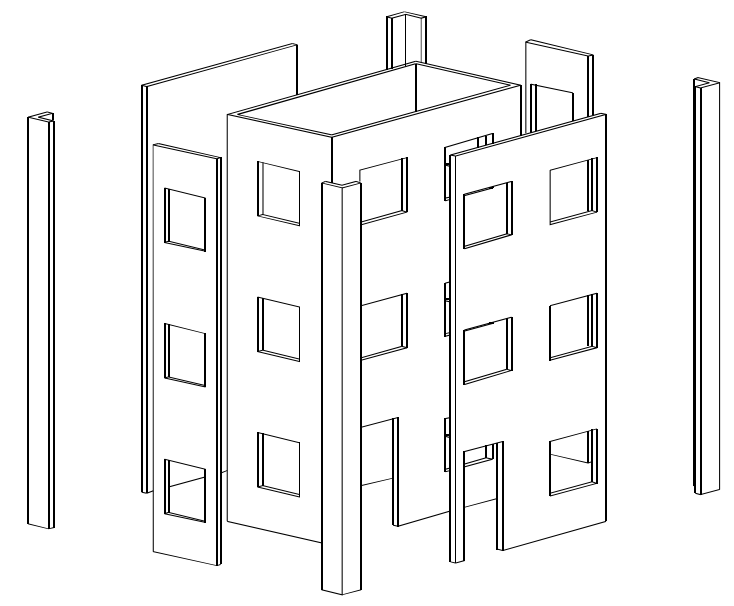

Figure 9. Facade division in corners and main faces.

The facade divisions can not cut forbidden zones, which are defined by elements like windows, doors, holes, etc. After the facade division in floors, the forbidden cutting zones are automatically identified for each floor.

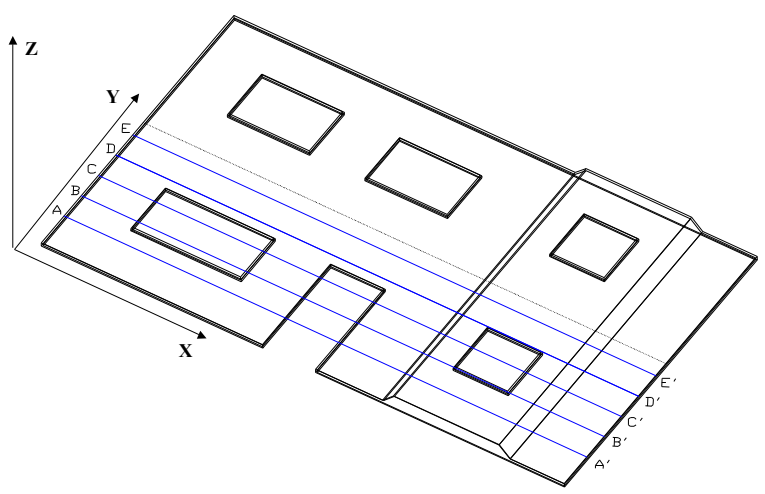

Figure 10 Definition of forbidden zones

Each floor is cut by a set of ZX parallel planes. These planes are evenly distributed on the Y-axis interval occupied by each floor, see figure 10 . Then the sections obtained by means of the cuts in $\mathrm{XZ}$ planes are analysed. At this point all discontinuities that appear in these sections are considered as forbidden zones.

There is a series of special cases, which could be encountered and are summarised in:

Very small holes: the solution is to adjust the distance between cutting planes according to the size of the holes.

Non rectangular windows (figure 11 to the left): this situation is solved considering the widest interval along $\mathrm{X}$-axis, as a forbidden zone that embraces the whole window.

Coincident windows: applying the previous approach to windows with any common interval, like 
those on figure 11 to the right. The obtained effect is to reduce the number of considered windows.
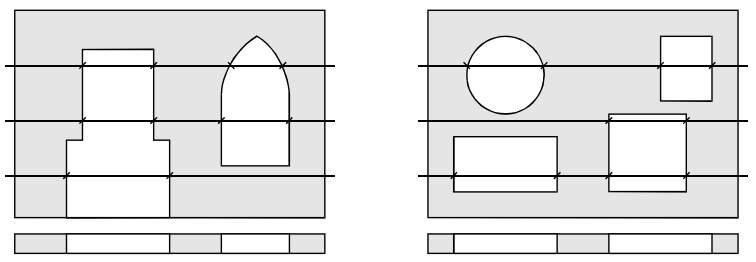

Figure 11 Special windows cases

\subsection{Criterions of facade partitions}

The final facade division is calculated satisfying the specifications. Algorithms have been developed according to three partition approaches:

To minimise the total number of panels in the facade: this criterion minimises the time transportation and the panels assembly.

To minimise the number of moulds: this criterion minimises the panels production time.

Mixed: to minimise the total number of panels trying to obtain the highest number of equal moulds.

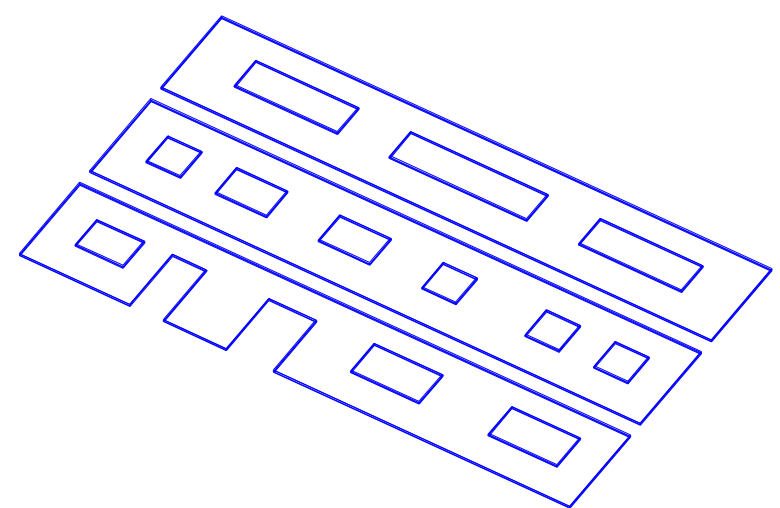

Figure 12 Main face divided in floors

The result of the previous phases is a horizontal divisions list for each main face and a vertical divisions list for each floor. The following procedures work with Boolean operations with threedimensional solids.

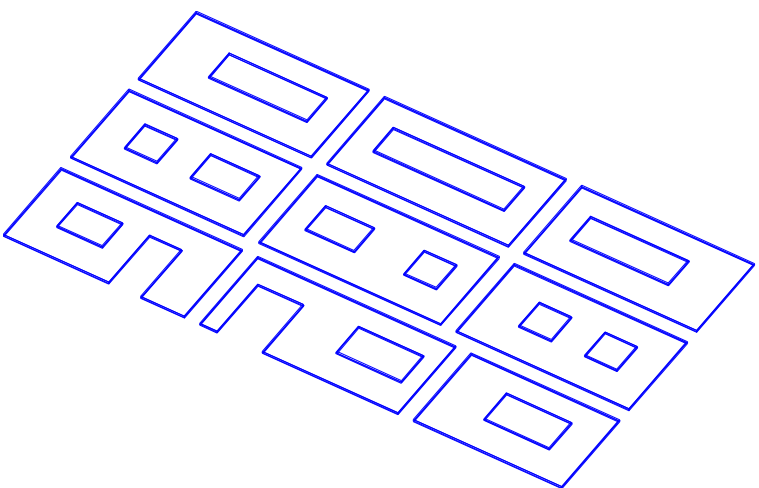

Fig. 13 Three dimensional solids of facade panels

With the horizontal divisions list the solid main face is divided into floors, as shown in figure 12 .
This figure shows the floors separated for better clarity of the drawing.

With the vertical divisions list each floor is divided into the solids that constitute it. Figure 13 shows a facade divided into all the panels that compose it.

\section{CONCLUSIONS}

The developed AUTMOD3 system presents a new tool to adapt traditional design to new construction technologies. The objective is to introduce modular design to obtain panels and modules that will be constructed in a factory and automatically assembled with robots.

The system was developed using a standard CAD software and the Object-Oriented language $\mathrm{C}++$. These characteristics provide features, which permit adding new functions to the system, in an easy way.

\section{REFERENCES}

[1] R. Diez, "Obtaining facade moulds to fabricate panels in with a CAD system", in Spanish, Licentiate Thesis, Polytechnic University of Madrid, 1996.

[2] E. Gambao, C. Balaguer, A. Barrientos, R. Saltarén and E. A. Puente, "Robot Assembly System for the Construction Process Automation", IEEE International Conference on Robotics \& Automation, Albuquerque (USA), 1997.

[3] R. Lewis, C. Séquin, "Generation of 3D building models from 2D architectural plans", Computer-Aided Design, vol.30, n 10, pp. 765-779, 1998.

[4] C. MacMahon, J. Browne, "CADCAM. From principles to practice", Addison-Wesley, 1993.

[5] J.M. Pastor, "Modular construction system oriented to robot by means of C.I.C. techniques", in Spanish, Ph.D. Thesis, Polytechnic University of Madrid, 1997.

[6] G. Pelliteri, "A tool for a first analysis of architectural façades", Automation in Construction, vol. $5, \mathrm{n}^{\circ} 5$, pp. 379-391, 1997

[7] L.F. Peñín; C. Balaguer; J.M. Pastor; F.J. Rodríguez; A. Barrientos; R. Aracil; "Robotized Spraying of Prefabricated Panels", IEEE Robotics \& Automation Magazine, Sept. 1998, pp. 18-29.

[8] O.Ransen, "AutoCAD programming in $\mathrm{C} / \mathrm{C}+$ +”, John Wiley \& Sons, 1997. 
[9] V. Karhu, "Product Model Based Design of Precast Facades", Licentiate Thesis, Royal Institute of Technology, Sweden, 1997. 